\title{
SÍNTOMAS SEUDO-PSICÓTICOS EN ADOLESCENTES DE LA POBLACIÓN GENERAL
}

\author{
Jordi E. OBiols ${ }^{1}$, MARCEla BARragÁN ${ }^{1}$, Jordi Vicens ${ }^{1}$, Y JOSÉ B. NAVARRO² \\ ${ }^{1}$ Unidad de Investigación en Psicopatología y Neuropsicología, Universidad Autónoma de Barcelona. \\ ${ }^{2}$ Departamento de Psicobiología y Metodología de las Ciencias de la Salud, Universidad Autónoma de Barcelona
}

\begin{abstract}
Resumen: Se han descrito experiencias psicóticas en poblaciones no clínicas. Estas experiencias pueden tener diferentes cursos evolutivos a largo plazo. La aparición de experiencias psicóticas ocasionales o síntomas seudo-psicóticos (SSP) en la población general abre posibilidades para comprender los mecanismos y factores de riesgo de la esquizotipia y de los trastornos del espectro esquizofrénico. El objetivo de este estudio es examinar la presencia de síntomas seudo-psicóticos (SSP) positivos, negativos y depresivos, en una muestra de 777 adolescentes de la población general utilizando para ello una escala autoadministrada. Los resultados hacen evidente la presencia de SSP en la muestra analizada. Las tres dimensiones de síntomas estuvieron significativamente correlacionadas entre sí. El aumento en los síntomas depresivos elevó las puntuaciones en las dimensiones positiva y negativa. Se encontraron diferencias de género en las puntuaciones de la dimensión depresiva.
\end{abstract}

Palabras clave: Síntomas seudo-psicóticos, adolescentes, población general

\section{Psychotic-like symptoms in adolescents of the general population}

\begin{abstract}
Psychotic-like experiences in non-clinical samples have been described. These experiences may evolve towards different outcomes in the long term ranging from social impairments to schizophrenia spectrum disorders. The emergence of occasional psychotic experiences or psychoticlike symptoms in the general population opens possibilities for understanding the mechanisms and risk factors for schizotypy and schizophrenic spectrum disorders. The purpose of this paper is to examine the prevalence of positive, negative and depressive psychotic-like symptoms in a sample of 777 adolescents of the general population, using a self-report scale. Results confirmed the presence of psychotic-like symptoms in the sample analyzed. All three dimensions of symptoms were significantly intercorrelated. An increase in depressive symptoms also increased positive and negative dimension scores. Gender differences were found in the depressive dimension scores.
\end{abstract}

Keywords: Psychotic-like symptoms, adolescents, general population.

\section{INTRODUCCIÓN}

El estudio de los síntomas seudo-psicóticos (SSP) en poblaciones no clínicas es importante, debido a su potencial para ampliar la comprensión de los factores de riesgo en las psicosis, cuya investigación ha estado limitada tradicionalmente a los sujetos con trastornos psicóticos (Meehl, 1962; Venables, 1995; Verdoux y Van Os, 2002). La aparición ocasional de estas experiencias en la población normal permite estudiarlas sin las desventajas derivadas

Recibido 5 febrero 2008; aceptado 8 mayo 2008

Correspondencia: Jordi E. Obiols, Campus de la UAB-Edifici B, 08193 Bellaterra (Cerdanyola del Vallès). Correo-e: Jordi.Obiols@uab.es de su exploración en pacientes psicóticos; es decir, sin los efectos de la medicación, la hospitalización y las dificultades de comunicación, a la vez que abren posibilidades de aclarar los mecanismos subyacentes de la esquizotipia e incluso de la esquizofrenia (McCreery y Claridge, 1996).

Actualmente, dentro del modelo dimensional de la psicopatología, se considera que los síntomas psicóticos son la expresión severa de rasgos presentes en la población general. Estos rasgos se manifiestan como variaciones observables entre los individuos, que van desde aquellos perfecta-

Agradecimientos: Este trabajo se llevó a cabo con el apoyo del Programa Alban, programa de becas de alto nivel de la Unión Europea para América Latina, beca n. ${ }^{\circ}$ E06D101876CO, y del Ministerio de Educación y Ciencia I+D (código BSO2003-05561/PSCE). 
mente ajustados hasta quienes, mostrando algunos signos de psicopatología, no son considerados clínicamente psicóticos (Peters, Joseph, Day y Garety, 2004). De esta manera, las formas transitorias o atenuadas de los síntomas mostrados por los individuos con psicosis, que no alcanzan el umbral clínico para requerir tratamiento, se denominan síntomas seudo-psicóticos (SSP) o signos esquizotípicos (Muela, García-León y Jiménez, 2007; Venables, 1995). Se ha sugerido que los SSP y la psicosis son fenómenos cuantitativa y no cualitativamente distintos, es decir, sus diferencias radican en la intensidad, frecuencia y nivel de malestar asociado, sin llegar a ser fenomenológicamente distintos (Yung et al., 2007).

El modelo dimensional de la psicosis implica un cambio respecto a la perspectiva categórica tradicional, que concebía los cuadros psicóticos como entidades dicotómicas y planteaba procesos de evaluación dirigidos a establecer si una persona presentaba o no una serie de síntomas (Kwapil, Chapman y Chapman, 1999). Este modelo es adecuado para la comprensión de los trastornos con una causa única, pero en el caso de la psicosis, cuya etiología es multifactorial, la distribución no sería completamente discontinua y dicotómica (Johns y Van Os, 2001). Por lo tanto, el concepto de continuum, enmarcado dentro del modelo dimensional, sería una represen- tación más adecuada de la variación en la salud mental de la población general (Van Os et al, 1999). En el modelo dimensional, se examina el grado en que la persona tiene una experiencia medida en varias dimensiones y no sólo la presencia o ausencia del síntoma (Hanssen et al., 2003). De este modo, los síntomas psicóticos se presentan en un rango de severidad, que depende de la frecuencia, duración, grado de implausibilidad o desviación, creencia en la experiencia y deterioro funcional (Kwapil et al., 1999).

\section{Investigación de los sintomas seudo-psicóti- cos en la población general}

A partir del modelo dimensional de la psicosis se han realizado estudios que identifican experiencias psicóticas en poblaciones no clínicas. El Netherlands Mental Health Survey and Incidence Study (NEMESIS), arrojó una prevalencia de SSP del 17,5\% en una muestra de la población general (Van Os, Hanssen, Bijl y Ravelli, 2000). En el National Survey of Mental Health and Wellbeing (NSMH) el 11,7\% de los participantes presentó algún SSP (Scott, Chant, Andrews y McGrath, 2006) y en el estudio de Mojtabai (2006) la prevalencia fue del 5,1\% (Tabla 1).

Tabla 1. Prevalencia de síntomas seudo-psicóticos en muestras no-clínicas

\begin{tabular}{|c|c|c|c|c|c|}
\hline Estudio & Año & $N$ & Tipo de Población & $\begin{array}{l}\text { Prevalencia } \\
\text { SSP }\end{array}$ & Otros resultados \\
\hline $\begin{array}{l}\text { Peters et al. } \\
\text { Delusions } \\
\text { Inventory PDI-40 } \\
\text { (Peters, Joseph } \\
\text { y Garety) }\end{array}$ & 1999 & $\begin{array}{l}272 \text { (muestra } \\
\text { no-clínica) } \\
20 \text { (muestra } \\
\text { clínica. }\end{array}$ & $\begin{array}{l}\text { Estudiantes universita- } \\
\text { rios adultos de } 19 \text { a } 75 \\
\text { años de edad. } \\
\text { Pacientes psicóticos de } \\
18 \text { a } 54 \text { años. }\end{array}$ & $25,2 \%$ & $\begin{array}{l}\text { Pacientes delirantes obtienen } \\
\text { puntuaciones superiores en ma- } \\
\text { lestar, preocupación y convic- } \\
\text { ción a las del grupo de la pobla- } \\
\text { ción general. }\end{array}$ \\
\hline $\begin{array}{l}\text { Netherlands Mental } \\
\text { Health Survey and } \\
\text { Incidence Study } \\
\text { NEMESIS } \\
\text { (Van Os et al.) }\end{array}$ & $\begin{array}{l}2000 \\
2001\end{array}$ & 7076 & $\begin{array}{l}\text { Población general de } 18 \\
\text { a } 64 \text { años de edad. }\end{array}$ & $17,5 \%$ & $\begin{array}{l}\text { Factores asociados: procedencia } \\
\text { urbana, edad juvenil, menores in- } \\
\text { gresos, menor nivel educativo, } \\
\text { desempleo, baja calidad de vida y } \\
\text { estado civil (soltero o divorciado) }\end{array}$ \\
\hline $\begin{array}{l}\text { Peters et al. } \\
\text { Delusions Inventory } \\
\text { PDI-21 } \\
\text { (Peters et al.) }\end{array}$ & 2004 & $\begin{array}{l}444 \text { (muestra } \\
\text { no-clínica) } \\
33 \text { (muestra } \\
\text { clínica) }\end{array}$ & $\begin{array}{l}\text { Población general de } 16 \\
\text { a } 67 \text { años de edad. } \\
\text { Pacientes delirantes de } \\
20 \text { a } 62 \text { años de edad. }\end{array}$ & $29,8 \%$ & $\begin{array}{l}\text { Pacientes delirantes obtienen pun- } \\
\text { tuaciones superiores en malestar } \\
\text { y preocupación a las de los indi- } \\
\text { viduos de la población general. }\end{array}$ \\
\hline
\end{tabular}


Tabla 1. Prevalencia de síntomas seudo-psicóticos en muestras no-clínicas (Continuación)

\begin{tabular}{|c|c|c|c|c|c|}
\hline Estudio & Año & $N$ & Tipo de Población & $\begin{array}{c}\text { Prevalencia } \\
\text { SSP }\end{array}$ & Otros resultados \\
\hline $\begin{array}{l}1997 \text { Australian } \\
\text { National Survey of } \\
\text { Mental Health and } \\
\text { Wellbeing } \\
\text { (Scott et al.) }\end{array}$ & 2006 & 10641 & $\begin{array}{l}\text { Población general ma- } \\
\text { yor de } 18 \text { años. }\end{array}$ & $11,7 \%$ & $\begin{array}{l}\text { Factores asociados: edad juvenil, } \\
\text { individuos solteros, separados } \\
\text { y divorciados, desempleados y } \\
\text { de zonas urbanas. }\end{array}$ \\
\hline $\begin{array}{l}2001 \text { US National } \\
\text { Household Survey on } \\
\text { Drug Abuse NHSDA } \\
\text { (Mojtabai, R). }\end{array}$ & 2006 & 38132 & $\begin{array}{l}\text { Población general ma- } \\
\text { yor de } 18 \text { años de edad. }\end{array}$ & $5,1 \%$ & $\begin{array}{l}2,2 \% \text { alucinaciones auditivas, } 2 \% \\
\text { visuales, } 1,4 \% \text { ideas de referencia, } \\
1 \% \text { experiencias de influencia, } \\
1 \% \text { ideación paranoide, } 0,8 \% \text { robo } \\
\text { de pensamientos y } 0,5 \% \text { pensa- } \\
\text { mientos insertados. }\end{array}$ \\
\hline (Loewy et al.) & 2007 & 1020 & $\begin{array}{l}\text { Estudiantes universita- } \\
\text { rios a partir de } 18 \text { años } \\
\text { de edad. }\end{array}$ & $35 \%$ & $\begin{array}{l}\text { Adjudicación de síntomas posi- } \\
\text { tivos es muy común }(93 \%) \text { entre } \\
\text { estudiantes. Es necesario incluir } \\
\text { criterios de frecuencia y males- } \\
\text { tar para mejorar especificidad. }\end{array}$ \\
\hline $\begin{array}{l}\text { Presente estudio } \\
\text { (Obiols et al.) }\end{array}$ & 2008 & 777 & $\begin{array}{l}\text { Adolescentes de la pobla- } \\
\text { ción general española. }\end{array}$ & $18,1 \%$ & $\begin{array}{l}\text { Aumento en síntomas depresi- } \\
\text { vos eleva sintomatología positi- } \\
\text { va y negativa. }\end{array}$ \\
\hline
\end{tabular}

Existe evidencia que sugiere que la psicosis puede ser entendida en términos de un continuum de variación en distintas dimensiones de síntomas independientes pero correlacionados. Stefanis et al. (2002), identificaron en la población general tres dimensiones de síntomas definidas en el cuestionario autoadministrado Community Assessment of Psychic Experiences (CAPE; Stefanis et al., 2002): síntomas positivos, negativos y depresivos. Además, encontraron que los síntomas positivos y negativos de la psicosis están asociados a experiencias de depresión en la población general y no sólo en muestras clínicas.

En este sentido, se ha sugerido que la continuidad del fenotipo psicótico se extiende desde la normalidad, pasa por los trastornos depresivos y llega a la psicosis clínica (Van Os, Hanssen, Bijl y Vollebergh, 2001). En la investigación de Van Os et al. (1999), un grupo de pacientes con síntomas de ansiedad y depresión obtuvo puntuaciones intermedias en el cuestionario $P e$ ters Delusions Inventory (PDI; Peters, Joseph y
Garety, 1999) respecto al grupo control y al grupo de sujetos con psicosis. Este resultado sugiere que la depresión podría ser un elemento favorecedor para el desarrollo de trastornos psicóticos (Verdoux et al., 1999). Otros estudios señalan que es frecuente la coexistencia de síntomas depresivos y psicóticos (Rosen, Miller, D'Andrea, McGlashan y Woods, 2006).

En cuanto a los tipos específicos de SSP descritos en la población general, Mojtabai (2007) encontró que los síntomas más frecuentes eran: alucinaciones auditivas $(2,2 \%)$, alucinaciones visuales $(2 \%)$, ideas de referencia $(1,4 \%)$, experiencias de influencia ( $1 \%)$, ideación paranoide $(1 \%)$, robo de pensamientos $(0,8 \%)$ y pensamientos insertados $(0,5 \%)$. Por su parte, Peters, Joseph y Garety (1999) encontraron puntuaciones elevadas en ideación delirante en el 10\% de una muestra de adultos de la población general.

Las personas con creencias espirituales o religiosas intensas son grupos de población no clínica en los que se han identificado experien- 
cias similares a la sintomatología psicótica. En el estudio de Peters, Day, McKenna y Orbach (1999) las personas pertenecientes a Nuevos Movimientos Religiosos NMR (Hare Krishnas y Druidas) no se diferenciaron de los pacientes delirantes en sus puntuaciones en el PDI ni en sus niveles de convicción. Sin embargo, el grupo NMR, presentó menor preocupación y malestar que los pacientes con delirios. Aunque la similitud en la frecuencia de adjudicación de los ítems del PDI podría llevar a concluir que el grupo NMR presentaba delirios, el análisis de las dimensiones de convicción, preocupación y malestar, sugiere que lo que hace que la gente cruce el umbral psicótico no es necesariamente el contenido sino las consecuencias de sus creencias.

Los hallazgos revisados hasta ahora indican que aún cuando el modelo categórico de la psicopatología haya ejercido influencia sobre el pensamiento y la investigación en psicosis, la evidencia acumulada en los últimos años sustenta la definición de la psicosis como un fenómeno dimensional, ubicado en un continuum junto a la normalidad (Verdoux y Van Os, 2002), 1levando al cuestionamiento de la definición dicotómica de la psicosis.

\section{Evolución de los síntomas seudo-psicóticos}

La investigación sobre los SSP en la población general ha ampliado el conocimiento disponible sobre su evolución y sus posibles implicaciones. Sin embargo, no hay conclusiones definitivas y el grado de riesgo que representan los SSP para la población no psicótica aún necesita más investigación (Yung, et al., 2007).

\section{Evolución no psicótica}

Algunas investigaciones indican que gran parte de los individuos con SSP nunca desarrollará trastornos psicóticos. En lugar de ello, permanecerán clínicamente compensados, pero con una mayor probabilidad de presentar manifestaciones clínicas leves, por ejemplo, cumpliendo criterios para trastornos del espectro esquizofrénico de menor severidad como la es- quizotipia (Gooding, Tallent y Matts, 2005). Los SSP se han asociado con una disminución en la calidad de vida (Svirskis et al., 2007), malestar emocional y comportamientos violentos (Mojtabai, 2006). Se ha encontrado que los SSP tienden a disminuir con la edad, pero de llegar a persistir, se asocian con deterioro social y del funcionamiento general (Rössler et al., 2007).

Hanssen, Bak, Bijl, Vollebergh y Van Os (2005) examinaron la incidencia y evolución de los SSP en un periodo de 2 años, en personas sin antecedentes de SSP. El 2\% de la muestra desarrolló SSP al cabo de 1 año y de este porcentaje, el $8 \%$ presentó una continuidad subclínica a los 2 años de seguimiento. Los sujetos con múltiples SSP tenían una mayor probabilidad de continuar presentándolos $(13,3 \%, 95 \% \mathrm{IC}=$ 12,3-14,4) que los sujetos con una sola experiencia $(6,9 \%, 95 \% \mathrm{IC}=6,1-7,7)$, cuyos síntomas tendieron a desaparecer. En términos de predicción a corto plazo, este estudio sugiere que la evolución más probable de los SSP es la discontinuidad o remisión. Aquellos que no remiten tienen una mayor probabilidad de permanecer como experiencias subclínicas, sin dar paso a trastornos psicóticos.

\section{Evolución hacia trastornos de tipo psicótico}

Se ha examinado el curso de los SSP y las características de las personas que evolucionan hacia trastornos psicóticos. En el estudio de Dunedin, Nueva Zelanda, Poulton et al. (2000) examinaron si las creencias delirantes y las experiencias alucinatorias a los 11 años de edad predecían una evolución esquizofrénica 15 años después. Los resultados mostraron una asociación entre el autoinforme de síntomas psicóticos infantiles y un posterior trastorno esquizofreniforme. Los niños con mayores índices de SSP, tenían una probabilidad 16 veces mayor, que los niños sin SSP de presentar un trastorno esquizofreniforme a los 26 años de edad.

No obstante, queda claro que no todas las personas con SSP desarrollan trastornos psicóticos. Por esta razón, Yung et al. (2006) estudiaron cuáles SSP tenían mayor probabilidad 
de evolucionar hacia un trastorno psicótico. Los resultados mostraron que los SSP que incluían experiencias extrañas e ideación persecutoria se asociaron con malestar, trastornos del estado de ánimo y del funcionamiento general. Por lo tanto, este tipo de SSP podría asociarse con un mayor riesgo para el desarrollo de trastornos psicóticos.

Ya se ha mencionado que los SSP incrementan el riesgo de desarrollar cuadros depresivos y que la presencia simultánea de depresión y SSP favorece el curso psicótico de estos últimos. Al respecto, se ha examinado la influencia de la depresión sobre los SSP (Yung et al., 2007), encontrándose que la disminución en el nivel de depresión se asocia con una reducción en el número de SSP, específicamente ideación persecutoria y experiencias extrañas. Por el contrario, la continuidad de los síntomas depresivos, se traduce en niveles significativamente más altos de SSP, lo que significa que la depresión es una variable importante para determinar la evolución de los SSP.

Al explicar el mecanismo por el cual la depresión afecta el curso de los SSP, se ha planteado que ésta podría incrementar la probabilidad de hacer evaluaciones negativas de las experiencias psicóticas lo que a su vez, aumentaría el malestar, la ansiedad, la depresión y el estrés percibido. Éste malestar puede generar cambios biológicos como el incremento en los niveles de cortisol, además del mantenimiento de los síntomas psicóticos, favoreciendo el establecimiento de un trastorno psicótico. De este modo, el tratamiento de la depresión en pacientes con SSP sería benéfico, no sólo al reducir el sufrimiento del individuo, sino disminuyendo los SSP y, por lo tanto, el riesgo de transición a un trastorno psicótico (Owens, Miller, Lawrie y Johnstone, 2005; Yung et al., 2007).

\section{Factores asociados a la aparición y mantenimiento de los síntomas seudo-psicóticos}

Las personas de la población general con SSP y los pacientes con psicosis, muestran similitudes en los aspectos relativos a la evolución de los síntomas. Por ejemplo, se ha encontrado que la edad afecta la expresión de delirios en los casos clínicos de psicosis y en sujetos de la población general (Verdoux y Van Os, 2002). En ambos casos, se ha observado una asociación negativa entre la edad y la manifestación de los síntomas (Scott et al., 2006). Así, los sujetos más jóvenes obtienen puntuaciones más altas en ideación persecutoria, alteraciones del pensamiento, grandiosidad y creencias paranormales (Rössler et al., 2007). La religiosidad es la única dimensión asociada positivamente con el aumento de la edad. Estos hallazgos sugieren que la adultez temprana es un periodo donde los SSP son más frecuentes y podría ser, junto con otros factores determinantes, una etapa evolutiva crítica para la expresión del rasgo de psicosis (Verdoux et al., 1998).

Se han descrito factores de riesgo de esquizofrenia que son también factores de vulnerabilidad para altos niveles de SSP. Algunos de estos factores incluyen bajo nivel educativo y baja calidad de vida (Van Os et al., 2000). La urbanicidad también ha sido identificada como una variable asociada con el incremento en los síntomas seudo-psicóticos y en los trastornos psicóticos (Scott et al., 2006; Van Os, Hanssen, de Graaf y Vollebergh, 2002; Van Os et al., 2001). Las personas residentes en centros urbanos, con mayor densidad de población, muestran niveles más altos de SSP en las escalas de creencias extrañas/pensamiento mágico y en pruebas de esquizotipia (Stefanis et al., 2004).

Finalmente, el estado civil ha sido asociado a la presencia de SSP. Estar casado o en una relación de hecho se relaciona con una disminución en la presencia de SSP (Mojtabai, 2007). Esta asociación ha sido explicada como el resultado de la menor probabilidad que tendrían los individuos con rasgos particulares de personalidad (esquizotípico, borderline, paranoide) para casarse o formar relaciones estables, aunque también se ha planteado que el malestar asociado al aislamiento social podría contribuir a la presencia de SSP (Scott et al., 2006).

El objetivo de este estudio fue examinar la presencia de SSP en una muestra de adolescentes de la población general española, utilizando para ello la escala autoadministrada 
CAPE. Se analizaron las tres dimensiones de síntomas: positiva, negativa y depresiva, definidas en la escala CAPE. Asimismo, se investigaron las asociaciones entre las tres dimensiones de la escala y el efecto de la sintomatología depresiva sobre los síntomas positivos y negativos. Se esperaba replicar los hallazgos de estudios previos realizados con muestras similares.

\section{MÉTODO}

\section{Participantes}

Participaron 927 adolescentes con edades comprendidas entre los 13 y 17 años, (468 chicas, 458 chicos y en un caso no se contó con esta información) estudiantes de secundaria en alguna de las 14 escuelas de la ciudad de Barcelona que aceptaron participar. De estas escuelas, nueve eran de carácter público y cinco eran privadas. El criterio de inclusión en el estudio fue: adecuada comprensión de lectura y manejo del idioma castellano como lengua materna. La participación en el estudio fue voluntaria y con previa autorización de los padres.

Para el análisis de datos se excluyeron 93 adolescentes provenientes de países distintos a España para evitar sesgos derivados de los diferentes bagajes culturales y asegurar un manejo homogéneo del idioma castellano. También se excluyeron los datos de 57 participantes con cuestionarios no diligenciados. De este modo, la muestra analizada incluye a 777 participantes (83,8\% de la muestra original). En el $50,9 \%$ de los casos fueron chicas $(n=396)$ y el promedio de edad del grupo fue de 14,44 (DT $=0,592)$. En el 63,7\% de los casos los adolescentes pertenecían a escuelas públicas y en el $36,3 \%$ a privadas.

\section{Instrumentos}

Community Assessment of Psychic Experiences CAPE (Stefanis et al., 2002). La escala CAPE fue desarrollada para evaluar auto-informes de experiencias psicóticas durante la vida, en los dominios afectivos y no afectivos. Evalúa tres dimensiones de síntomas: positivos, negativos y depresivos, y mide en una escala dimensional la frecuencia de estas experiencias. Cada pregunta se responde eligiendo una de las posibilidades presentadas en una escala de 4 puntos que abarca desde nunca (1) hasta casi siempre (4). Consta de 42 ítems que evalúan las de dimensiones positiva y negativa y de los síntomas psicóticos y los síntomas depresivos (Stefanis et al., 2002). En la versión en castellano se incluyen tres ítems que evalúan sinceridad. La escala CAPE ha mostrado una adecuada validez y fiabilidad. Estudios de seguimiento que evalúan sus propiedades psicométricas han arrojado tamaños de efecto altos para la estabilidad interna $(0,6-0,8)$, indicando que las dimensiones de experiencias subclínicas muestran una fuerte asociación con las mismas dimensiones en casos de seguimiento. Dadas sus propiedades y que su aplicación es económica y efectiva, su uso se ha recomendado en la investigación con la población general (Konings, Bak, Hanssen, Van Os y Krabbendam, 2006). El estudio local de validación arrojó un a de Cronbach de 0,89 para la escala global y de $0,84,0,78$ y 0,79 para la dimensión positiva, negativa y depresiva respectivamente.

\section{Procedimiento}

Las 14 escuelas de Barcelona fueron elegidas a través de un muestro estratificado y conforman una muestra representativa de las escuelas de esta ciudad. Se solicitó a los equipos directivos de estos centros educativos autorización para aplicar colectivamente, en las aulas de clase, la prueba CAPE. Asimismo, se solicitó consentimiento a los padres y a los participantes.

\section{Análisis de datos}

Tras verificar la normalidad de la distribución de las puntuaciones del CAPE se utilizó la prueba $t$ de student para determinar la presencia de diferencias significativas entre la puntuación total, las dimensiones del CAPE y el 
género de los participantes. Asimismo, se realizó un análisis correlacional de Pearson entre la edad de los participantes y las puntuaciones en la prueba. Se estableció el percentil 25 y el 75 como puntos de corte para definir las puntuaciones bajas y altas.

\section{RESULTADOS}

La Tabla 2 incluye los índices estadísticos descriptivos para las tres dimensiones y para la puntuación total del CAPE. No se encontraron diferencias significativas en la puntuación total del CAPE asociadas a la edad $(r=0,025 ; p$ $=0,494)$. Las diferencias asociadas al género, fueron prácticamente significativas (femenino: $M=66,85 ; D T=13,309$; masculino $M=65,08$; $D T=12,614 ; t=1,904 ; p=0,057)$. El análisis de cada una de las dimensiones del CAPE, no arrojó diferencias significativas asociadas a la edad (positiva $r=0,20 ; p=0,585$; negativa $r=$ 0,$026 ; p=0,464$; depresiva $r=0,014 ; p=0,694$ ). En cuanto al género, las chicas mostraron puntuaciones significativamente más elevadas en la dimensión depresiva en comparación con los chicos (Tabla 3). Las dimensiones de síntomas del CAPE estuvieron significativamente correlacionadas entre sí (Tabla 4) y el aumento en la sintomatología depresiva se asoció con un incremento en las puntuaciones en las dimensiones positiva y negativa (Figura 1).

Tabla 2. Distribución de las dimensiones positiva, negativa y depresiva del CAPE

\begin{tabular}{lcccccc}
\hline \multicolumn{1}{c}{$\begin{array}{c}\text { Dimensión } \\
\text { CAPE }\end{array}$} & Media & DT & Mediana & Mínimo & Máximo & $\begin{array}{c}\text { Puntuación } \\
\text { máxima posible }\end{array}$ \\
\hline Positiva & 29,50 & 6,66 & 28 & 20 & 62 & 80 \\
Negativa & 23,82 & 5,20 & 23 & 14 & 49 & 56 \\
Depresiva & 12,67 & 3,68 & 12 & 7 & 27 & 32 \\
Total & 65,98 & 12,99 & 64 & 43 & 136 & 168 \\
\hline
\end{tabular}

Nota: $\mathrm{CAPE}=$ Community Assessment of Psychic Experiences.

Tabla 3. Puntuación en las dimensiones del CAPE según género: comparación de medias

\begin{tabular}{|c|c|c|c|c|c|c|c|}
\hline \multirow{2}{*}{ Dimensión } & & \multicolumn{2}{|c|}{ Género } & \multirow[b]{2}{*}{$t$} & \multirow[b]{2}{*}{$p$} & \multirow{2}{*}{$\begin{array}{l}\text { Diferencia } \\
\text { de medias }\end{array}$} & \multirow{2}{*}{$\begin{array}{c}\text { IC 95\% } \\
\text { Diferencia } \\
\text { de medias }\end{array}$} \\
\hline & & Femenino & Masculino & & & & \\
\hline Positiva & $\begin{array}{l}\text { Media } \\
D T\end{array}$ & $\begin{array}{r}29,65 \\
6,95\end{array}$ & $\begin{array}{r}29,33 \\
6,37\end{array}$ & 0,670 & 0,503 & 0,321 & $-0,619$ a 1,260 \\
\hline Negativa & $\begin{array}{l}\text { Media } \\
D T\end{array}$ & $\begin{array}{c}23,68 \\
5,11\end{array}$ & $\begin{array}{c}23,98 \\
5,30\end{array}$ & $-0,732$ & 0,464 & $-0,274$ & $-1,007$ a 0,460 \\
\hline Depresiva & $\begin{array}{l}\text { Media } \\
D T\end{array}$ & $\begin{array}{c}13,52 \\
3,89\end{array}$ & $\begin{array}{c}11,79 \\
3,23\end{array}$ & 6,714 & 0,000 & 1,725 & 1,221 a 2,229 \\
\hline
\end{tabular}

Nota $:$ CAPE $=$ Community Assessment of Psychic Experiences.

Tabla 4. Correlaciones entre las puntuaciones en las dimensiones del CAPE

\begin{tabular}{lccc}
\hline \multicolumn{1}{c}{ Dimensión CAPE } & Positiva & Negativa & Depresiva \\
\hline Positiva & 1 & $0,540^{* *}$ & $0,494^{* *}$ \\
Negativa & & 1 & $0,576^{* *}$ \\
Depresiva & & & 1 \\
\hline
\end{tabular}

Nota $:$ CAPE $=$ Community Assessment of Psychic Experiences. $* * p<0,01$. 
Los 42 ítems de la prueba fueron respondidos de manera afirmativa («algunas veces», «a menudo», «casi siempre») al menos en una ocasión. El 51,1\% de los participantes dio la res-

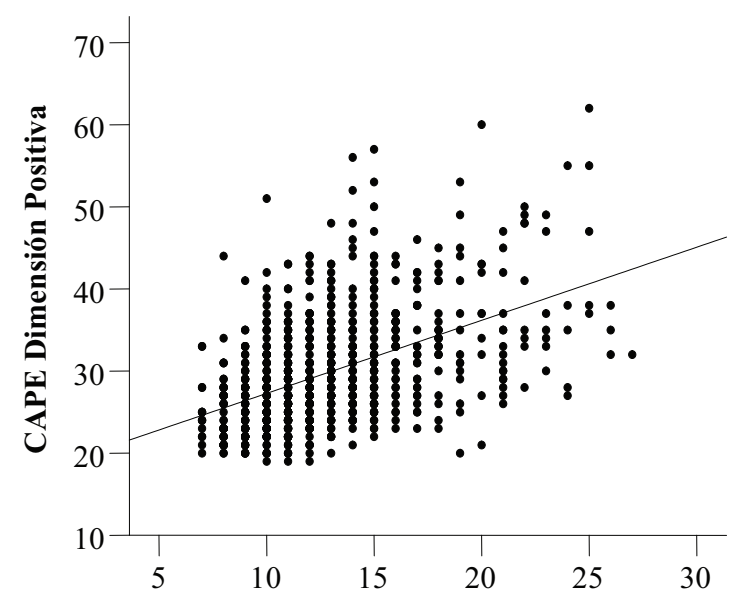

CAPE Dimensión Depresiva puesta «casi siempre» por lo menos a un ítem de la prueba. En la Tabla 5 se presenta el número de ítems referidos por los participantes en la escala global (CAPE total) y en cada dimensión.

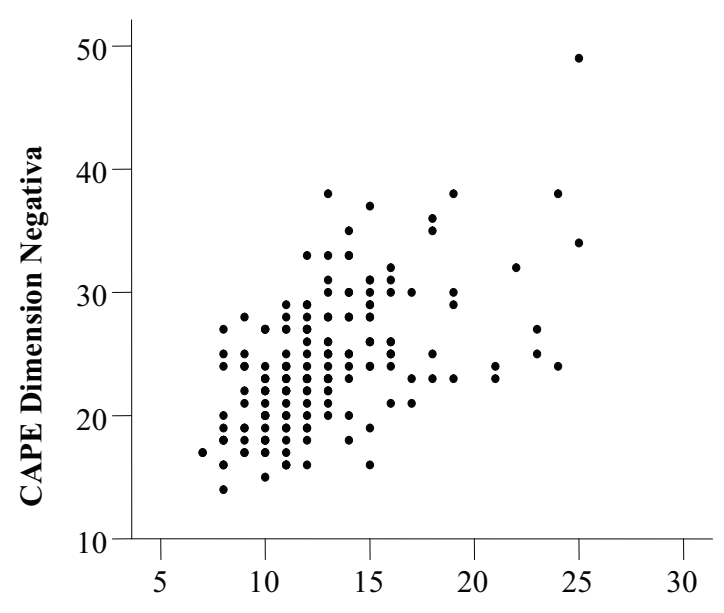

CAPE Dimensión Depresiva

Figura 1. Dimensiones positiva y negativa del CAPE según puntuación en la dimensión depresiva (CAPE $=$ Community Assessment of Psychic Experiences).

Tabla 5. Número de ítems del CAPE referidos por los participantes según dimensión (positiva, negativa, depresiva y CAPE total) y frecuencia de presentación («algunas veces», «a menudo» y «casi siempre»)

\begin{tabular}{|c|c|c|c|c|c|c|c|c|}
\hline \multirow{3}{*}{ Frecuencia } & \multicolumn{8}{|c|}{ Número de items } \\
\hline & \multicolumn{2}{|c|}{0} & \multicolumn{2}{|c|}{1} & \multicolumn{2}{|c|}{2} & \multicolumn{2}{|c|}{$\geq 3$} \\
\hline & $\%$ & $n$ & $\%$ & $n$ & $\%$ & $n$ & $\%$ & $N$ \\
\hline \multicolumn{9}{|l|}{ Dimensión Positiva } \\
\hline Casi siempre & 60,7 & 472 & 22,7 & 176 & 9,1 & 71 & 7,5 & 58 \\
\hline A menudo & 25,4 & 197 & 24,2 & 188 & 20,1 & 156 & 30,3 & 236 \\
\hline Algunas veces & 1 & 8 & 3,6 & 28 & 9,5 & 74 & 85,9 & 667 \\
\hline \multicolumn{9}{|l|}{ Dimensión Negativa } \\
\hline Casi siempre & 78,1 & 607 & 14,2 & 110 & 3,7 & 29 & 4 & 31 \\
\hline A menudo & 41,4 & 322 & 22,5 & 175 & 15,6 & 121 & 20,5 & 159 \\
\hline Algunas veces & 2,3 & 18 & 4 & 31 & 6,4 & 50 & 87,3 & 678 \\
\hline \multicolumn{9}{|l|}{ Dimensión Depresiva } \\
\hline Casi siempre & 83,9 & 652 & 8,5 & 66 & 3,1 & 24 & 4,5 & 35 \\
\hline A menudo & 58,6 & 455 & 21,9 & 170 & 10 & 78 & 9,5 & 74 \\
\hline Algunas veces & 4,5 & 35 & 12,4 & 96 & 16,5 & 128 & 66,6 & 518 \\
\hline \multicolumn{9}{|l|}{ CAPE Total } \\
\hline Casi siempre & 48,9 & 380 & 24,6 & 191 & 8,4 & 65 & 18,1 & 141 \\
\hline A menudo & 13,4 & 104 & 15,1 & 117 & 12,7 & 99 & 58,8 & 457 \\
\hline Algunas veces & 4,5 & 2 & 0 & 0 & 0,9 & 7 & 98,3 & 764 \\
\hline
\end{tabular}

Nota: $\mathrm{CAPE}=$ Community Assessment of Psychic Experiences. 
Para el análisis de la dimensión positiva, se seleccionaron diez ítems que describen las alteraciones del pensamiento y la percepción más características de los trastornos psicóticos. El total de respuestas afirmativas a estos ítems, es decir que muestran alguna presencia del síntoma, evidencia una prevalencia elevada de ideación persecutoria $(58 \%)$, pensamiento mágico $(34,3 \%)$ y experiencias alucinatorias tales como oír voces $(31,8 \%)$ y voces hablando entre ellas $(19,8 \%)$. Al analizar la frecuencia de presentación de estos síntomas, se observa que un 18,1\% de la muestra refiere una frecuencia alta («casi siempre») al menos para un síntoma positivo, de este porcentaje el $51,8 \%$ pertenece al géne- ro masculino. En el caso de la dimensión negativa, se analizaron 7 de los 14 ítems de la subescala, que describen con mayor claridad los síntomas negativos característicos de la psicosis (abulia, aplanamiento afectivo y anhedonia). El 16,1\% de la muestra refirió alguno de los síntomas seleccionados con una frecuencia alta («casi siempre»). Se encontró una tendencia ligeramente mayor en los chicos a presentar este tipo de síntomas (52,8\% respecto a las chicas). Finalmente, en la dimensión depresiva, el 16\% de la muestra se adjudicó síntomas de esta dimensión con una frecuencia alta, de este porcentaje el $66,4 \%$ pertenece al género femenino (Tabla 6).

Tabla 6. Distribución total y por género de los ítems seleccionados

(positivos, negativos y depresivos) del CAPE con una frecuencia máxima de presentación («casi siempre»)

\begin{tabular}{|c|c|c|c|}
\hline \multirow[b]{2}{*}{ Ítem } & \multicolumn{3}{|c|}{ Género } \\
\hline & $\begin{array}{c}\text { Femenino } \\
n(\%) \\
(N=396) \\
\end{array}$ & $\begin{array}{c}\text { Masculino } \\
n(\%) \\
(N=381) \\
\end{array}$ & $\begin{array}{c}\text { Total } \\
n(\%) \\
(N=777)\end{array}$ \\
\hline \multicolumn{4}{|l|}{ Síntomas Positivos } \\
\hline - ¿Piensas que los aparatos eléctricos pueden influir en tu forma de pensar? & $5(1,26)$ & $21(5,51)$ & $26(3,34)$ \\
\hline $\begin{array}{l}\text { - ¿Tienes la impresión de que alguna noticia de la prensa, radio o televisión se } \\
\text { refiere o está exclusivamente dirigida a ti? }\end{array}$ & $4(1,01)$ & $5(1,31)$ & $9(1,15)$ \\
\hline - ¿Oyes voces cuando estas solo? & $15(3,78)$ & $6(1,57)$ & $21(2,70)$ \\
\hline - ¿Oyes voces hablando entre ellas cuando estas solo? & $6(1,51)$ & $2(0,52)$ & $8(1,02)$ \\
\hline - ¿Ves cosas, personas o animales que otras personas no pueden ver? & $8(2,02)$ & $3(0,78)$ & $11(1,42)$ \\
\hline - ¿Sientes que te siguen de alguna forma o «andan detrás de ti»? & $11(2,77)$ & $9(2,36)$ & $20(2,57)$ \\
\hline - ¿Tienes la impresión de que hay un complot contra ti? & $6(1,51)$ & $14(3,67)$ & $20(2,57)$ \\
\hline - ¿Has sentido como si te estuvieran sacando los pensamientos de la cabeza? & $4(1,01)$ & $5(1,31)$ & $9(1,15)$ \\
\hline - ¿Has tenido pensamientos tan intensos que pensaste que los demás podían oírlos? & $6(1,51)$ & $3(0,78)$ & $9(1,15)$ \\
\hline $\begin{array}{l}\text { - ¿Has creído que un miembro de tu familia, amigo o conocido ha sido reemplazado } \\
\text { por un doble? }\end{array}$ & $3(0,75)$ & $5(1,31)$ & $8(1,02)$ \\
\hline \multicolumn{4}{|l|}{ Síntomas Negativos } \\
\hline - ¿Sientes que te faltan ganas para hacer las cosas? & $15(3,78)$ & $22(5,77)$ & $37(4,76)$ \\
\hline - ¿Piensas que te emocionas poco o casi nada en situaciones importantes? & $16(4,04)$ & $17(4,46)$ & $33(4,25)$ \\
\hline - ¿Has pensado que estás descuidando tu aspecto físico o cuidado personal? & $13(3,28)$ & $8(2,09)$ & $21(2,70)$ \\
\hline - ¿Sientes que tus emociones son poco intensas? & $4(1,01)$ & $7(1,83)$ & $11(1,42)$ \\
\hline - ¿Sientes que te falta energía? & $7(1,76)$ & $2(0,52)$ & $9(1,15)$ \\
\hline - ¿Sientes que no te interesa estar con otras personas? & 0 & $7(1,83)$ & $7(0,90)$ \\
\hline - ¿Sientes que tu mente está vacía? & $4(1,01)$ & $3(0,78)$ & $7(0,90)$ \\
\hline \multicolumn{4}{|l|}{ Síntomas Depresivos } \\
\hline - ¿Te sientes pesimista (como si todo fuera a ir mal? & $34(8,58)$ & $19(4,98)$ & $53(6,82)$ \\
\hline - ¿Lloras sin motivo? & $33(8,33)$ & $4(1,05)$ & $37(4,76)$ \\
\hline - ¿Te sientes fracasado? & $23(5,80)$ & $13(3,41)$ & $36(4,63)$ \\
\hline - ¿Piensas que no quieres vivir más? & $24(6,06)$ & $11(2,88)$ & $35(4,50)$ \\
\hline - ¿Te sientes triste? & $28(7,07)$ & $3(0,78)$ & $31(3,98)$ \\
\hline - ¿Piensas que no tienes futuro? & $17(4,29)$ & $14(3,67)$ & $31(3,99)$ \\
\hline - ¿Te sientes culpable? & $20(5,05)$ & $5(1,31)$ & $25(3,21)$ \\
\hline
\end{tabular}

Nota: CAPE $=$ Community Assessment of Psychic Experiences. 


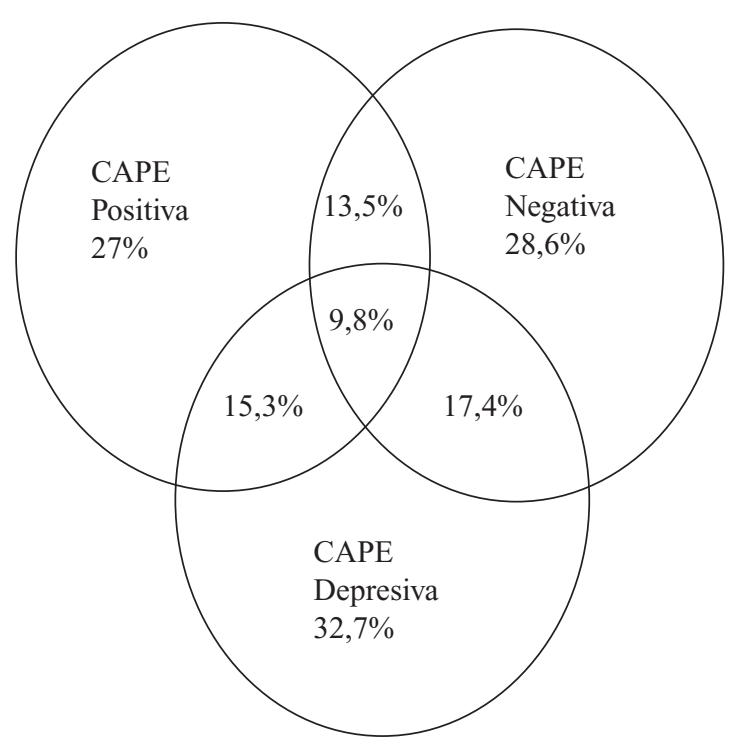

Figura 2. Puntuaciones ubicadas por encima del percentil 75 en cada dimensión del CAPE $\mathrm{y}$ en las intersecciones derivadas de combinar las distintas dimensiones $(n=76)$. CAPE $=($ Community Assessment of Psychic Experiences).

Por otra parte, al explorar las puntuaciones extremas en la muestra analizada, se encontró un grupo de 85 adolescentes $(10,9 \%)$ con puntuaciones situadas por debajo del percentil 25 en las tres dimensiones y un total de 76 participantes $(9,8 \%)$ con puntuaciones altas en las tres dimensiones, es decir, ubicadas por encima del percentil 75. En la Figura 2 se presenta la distribución de las puntuaciones altas en cada dimensión del CAPE y para cada combinación de dimensiones.

\section{DISCUSIÓN}

El objetivo de este estudio fue examinar la presencia de SSP en adolescentes de la población general española. El análisis de las puntuaciones en la prueba auto-administrada CAPE, obtenidas por 777 adolescentes, permitió encontrar evidencia de las dos dimensiones de experiencias características de los trastornos psicóticos: síntomas positivos, negativos y síntomas depresivos. La presencia de estos síntomas en la muestra analizada, confirma hallazgos previos que sugerían que los síntomas de psicosis se distribuyen en la población general, y que la psicosis puede concebirse como un continuum de variación en distintas dimensiones correlacionadas de síntomas (Stefanis et al., 2002; Van Os et al., 2001).

Las tres dimensiones de síntomas estuvieron correlacionadas de manera significativa, es decir, los jóvenes con SSP tendieron a manifestar sintomatología positiva, negativa y depresiva de manera simultánea, en lugar de un único síntoma o dimensión aislada de síntomas. Esta coexistencia de síntomas, como plantean Hanssen et al. (2005), aumenta el riesgo de evolucionar hacia trastornos psicóticos o de que estos síntomas sean continuos en el tiempo. La continuidad de los SSP, aún sin dar lugar a trastornos psicóticos como tal, tiene relevancia clínica dado que se ha asociado con dificultades sociales, alteraciones en el funcionamiento general (Rössler et al., 2007) y conductas violentas (Mojtabai, 2007). En este estudio, 397 adolescentes manifestaron por lo menos un SSP con una frecuencia alta. De este grupo, a la luz de los hallazgos previamente citados, se podría plantear la hipótesis de que el 24,6\% (que refirió un único síntoma) tendería a la remisión, mientras el 18,1\% (que manifestó tres o más SSP) y especialmente el $9,8 \%$ de los participantes con puntuaciones altas en las tres dimensiones, presentaría un riesgo mayor para el desarrollo de trastornos psicóticos o bien para la continuidad de estas experiencias con las consecuencias psicosociales que ello implicaría.

El estudio de SSP en muestras de la población general ha arrojado tasas de prevalencia que oscilan entre el 5,1\% (Mojtabai, 2007) y el $35 \%$ (Loewy, Johnson y Cannon, 2007). En este estudio, el criterio para estimar la prevalencia de SSP, fue referir al menos tres síntomas de la prueba CAPE con la máxima intensidad («casi siempre»). Se encontró una prevalencia de $18,1 \%$, resultado que se encuentra dentro de los rangos referidos en estudios previos con muestras de la población general (Tabla 1).

Los SSP se asocian con una edad juvenil y con un entorno urbano (Rössler et al., 2007; 
Scott et al., 2006; Van Os et al., 2001). De este modo, la relativamente alta prevalencia de múltiples SSP puede explicarse en parte por las características de nuestra muestra (edad adolescente y procedencia urbana). La etapa del ciclo evolutivo de los participantes permite contemplar la posibilidad de remisión de los síntomas. Se ha descrito la procedencia urbana, como un factor de riesgo ambiental que desde edades tempranas genera una vulnerabilidad general. Esta vulnerabilidad se puede reflejar en rasgos positivos o negativos de psicosis sin que necesariamente dé lugar a un trastorno psicótico (Van Os et al., 2002). Es decir, la multiplicidad de síntomas podría estar anticipando su continuidad y aunque no evolucionen hacia una psicosis franca sí podrían tener efectos negativos sobre la calidad de vida de los jóvenes.

Se encontraron algunas diferencias de género en la distribución de los SSP. Aunque las diferencias encontradas para la puntuación total del CAPE no fueron significativas si permiten observar que las chicas tendieron a presentar puntuaciones generales más altas que las obtenidas por los chicos. El análisis de las dimensiones del CAPE mostró que las chicas obtuvieron puntuaciones más elevadas que los chicos en los ítems que evalúan síntomas depresivos. Este resultado es consistente con hallazgos que revelan diferencias entre hombres y mujeres en escalas que evalúan depresión, donde las adolescentes de género femenino muestran sintomatología depresiva significativamente mayor a la evidenciada por los adolescentes de género masculino (Santos, Richards y Bleckley, 2007).

La comparación de las dimensiones positiva y negativa según el género de los participantes no arrojó diferencias significativas, aunque algunos ítems de estas subescalas si reflejan ciertas tendencias. En la dimensión positiva, las chicas tendieron a referir experiencias alucinatorias en mayor medida que los chicos, mientras los chicos obtuvieron puntuaciones superiores en los ítems que evalúan ideación delirante. Estos resultados son parcialmente consistentes con los obtenidos por Van Os et al. (2000), quienes encontraron una asociación significativa entre el género femenino y la presencia tanto de deli- rios como de alucinaciones. Otros estudios no muestran asociación entre SSP y género ( $\mathrm{Scott}$ et al., 2006).

En la dimensión negativa, los chicos presentaron puntuaciones más altas, en la mayor parte de los síntomas a excepción de los ítems «sientes que estas descuidando tu aspecto físico o cuidado personal» $\mathrm{y}$ «sientes que te falta energía» referidos con mayor frecuencia por mujeres. Estos dos ítems pueden estar reflejando, en el caso de las chicas que obtuvieron puntuaciones altas en la dimensión depresiva, síntomas adicionales de depresión. Otros estudios también muestran resultados que describen un solapamiento entre los síntomas negativos y depresivos (Häfner, Maurer, Trendler, an der Heiden y Schmidt, 2005). Por su parte, el ítem que alude al desinterés por las relaciones interpersonales y que podría estar evidenciando signos de aislamiento social fue referido exclusivamente por chicos (con una frecuencia de «casi siempre»). Este síntoma se ha asociado con un alto riesgo para la continuidad de los SSP y con una evolución psicótica. Respecto a esta asociación se ha planteado que el aislamiento social podría estar reflejando rasgos de personalidad que dificultarían el establecimiento de vínculos y relaciones estables, dificultad que ha sido señalada como factor de riesgo para el mantenimiento de los SSP y para el desarrollo de trastornos del espectro psicótico (Scott et al., 2006).

Los resultados de este estudio muestran un incremento en los síntomas positivos y negativos en la medida en que se elevan las puntuaciones en la dimensión depresiva: los adolescentes con mayor sintomatología depresiva, presentaron puntuaciones más altas en las otras dimensiones (Figura 1). Este hallazgo es consistente con los planteamientos de autores como Stefanis et al. (2002), quienes proponen que la experiencia de depresión acompaña los rasgos positivos y negativos de psicosis en la población general y no exclusivamente en muestras clínicas. Además, a la luz de hallazgos previos que sugieren un vínculo entre depresión y trastornos psicóticos (Häfner et al., 2005; Verdoux et al., 1999; Yung et al., 2007), los resultados podrían estar señalando una mayor vulnerabilidad a la psicosis entre los jóvenes que obtuvieron puntuacio- 
nes elevadas en la dimensión depresiva.

Es necesario señalar algunas limitaciones de este estudio. Los resultados fueron obtenidos exclusivamente a partir de un auto-informe y no se presentan datos de otra fuente de validez externa como sería una entrevista clínica u otros instrumentos. Por esta razón, los datos sobre la prevalencia de SSP presentados en este trabajo podrían no ser los reales. De la misma manera, no es posible determinar la relación entre las respuestas del CAPE y la presencia de trastornos psicóticos o depresivos en los participantes. Por lo tanto, los datos obtenidos y presentados aquí constituyen datos de prevalencia aproximados de SSP en adolescentes de la población general y sin alteraciones conocidas o diagnósticos de trastornos psicóticos.

Este estudio es de tipo transversal de manera que no se presentan datos de seguimiento sobre la evolución de los SSP para muestras de la población española, al igual que las características de los participantes y de los SSP en relación a un determinado curso longitudinal. Por esta razón, en el futuro sería importante llevar a cabo estudios de tipo longitudinal. Esto con el fin de complementar los datos transversales obtenidos aquí y aclarar el papel de los SSP como factores de riesgo o vulnerabilidad para los trastornos psicóticos.

Podemos concluir que la prevalencia puntual de SSP del 18,1\% encontrada en una muestra de adolescentes de la población general española, se encuentra en consonancia con los resultados de estudios previos que describen la presencia de estos síntomas en la población general. Estos hallazgos apoyan el modelo de continuum psicótico, donde se propone que los síntomas de psicosis están presentes en personas de la población general aunque con ciertas diferencias respecto a los pacientes con trastornos psicóticos. Asimismo, las tres dimensiones de síntomas estuvieron correlacionadas entre sí de manera significativa y los síntomas depresivos ejercieron influencia sobre las puntuaciones en las dimensiones de síntomas positivos y negativos. De este modo, un incremento en el nivel de depresión se vio acompañado por un aumento tanto en la sintomatología positiva como en la negativa.

\section{REFERENCIAS}

Gooding, D., Tallent, K., y Matts, C. (2005). Clinical status of at.risk individuals 5 years later: further evaluation of the psychometric high-risk strategy. Journal of Abnormal Psychology, 114, 170-175.

Häfner, H., Maurer, K., Trendler, G., an der Heiden, W., y Schmidt (2005). The early course of schizophrenia and depression. European Archives of Psychiatry and Clinical Neuroscience, 255, 167-173.

Hanssen, M., Bak, M., Bijl, R., Vollebergh, W., y Van Os, J. (2005). The incidence and outcome of subclinical psychotic experiences in the general population. The British Journal of Clinical Psychology, 44, 181-191.

Hanssen, M., Peeters, F., Krabbendam, L., Radstake, S., Verdoux, H., y Van Os, J. (2003). How psychotic are individuals with non-psychotic disorders? Social Psychiatry and Psychiatric Epidemiology, 38, 149-154.

Johns, L., y Van Os, J. (2001). The continuity of psychotic experiences in the general population. Clinical Psychology Review, 21, 1125-1141.

Konings, M., Bak, M., Hanssen, M., Van Os, J., y Krabbendam, L. (2006). Validity and reliability of the CAPE: a self-report instrument for the measurement of psychotic experiences in the general population. Acta Psychiatrica Scandinavica, 114, 55-61.

Kwapil, T., Chapman, L., y Chapman, J. (1999). Validity and usefulness of the Wisconsin manual for assessing psychotic-like experiences. Schizophrenia Bulletin, 25, 363-375.

Loewy, R., Johnson, J., y Cannon, T. (2007). Self-report of attenuated psychotic experiences in a college population. Schizophrenia Research, 93, 144-151.

McCreery, C., y Claridge, G. (1996). A study of hallucination in normal subjects-1. Self-report data. Personality and Individual Differences, 21, 739-747.

Meehl, P. (1962). Schizotaxia, schizotypy, schizophrenia. American Psychologist, 17, 827-838.

Mojtabai, R. (2006). Psychotic-like experiences and interpersonal violence in the general population. Social Psychiatry and Psychiatric Epidemiology, 41, 183-190.

Muela, J.A., García-León, A., y Jiménez, M.D. (2007). Relación de la esquizotipia psicométrica con variables emocionales y socioambientales. Revista de Psicopatología y Psicología Clínica, 12, 213-214.

Owens, D., Miller., Lawrie, S., y Johnstone, E. (2005). Pathogenesis of schizophrenia: a psychopathological perspective. British Journal of Psychiatry, 186, 386-393.

Peters, E., Day, S., McKenna, J., y Orbach, G. (1999). Delusional ideation in the religious and psychotic populations. British Journal of Clinical Psychology, 38, 8396.

Peters, E., Joseph, S., Day, S., y Garety, P. (2004). Measuring delusional ideation: the 21-item Peters et al. De- 
lusions inventory (PDI). Schizophrenia Bulletin, 30, 1005-1022.

Peters, E., Joseph, S., y Garety, P. (1999). Measurement of delusional ideation in the normal population: introducing the PDI (Peters et al. Delusions Inventory). Schizophrenia Bulletin, 25, 553-576.

Poulton, R., Caspi, A., Moffit, T., Cannon, M., Murray, R., y Harrington, H. (2000). Children`s self-reported psychotic symptoms and adult schizopreniform disorder. A 15-year longitudinal study. Archives of General Psychiatry, 57, 1053-1058.

Rosen, J., Miller, T., D`Andrea, J., McGlasahn, T., y Woods, S. (2006). Comorbid diagnoses in patients meeting criteria for the schizoprenia prodrome. Schizophrenia Research, 85, 124-131.

Rössler, W., Riecher-Rössler, A., Angst, J., Murray, R., Gamma, A., Eich, D., Van Os, J., y Gross, V. (2007). Psychotic experiences in the general population: a twenty-year prospective community study. Schizophrenia Research, 92, 1-14.

Santos, M., Richards, S., y Bleckley, K. (2007). Comorbidity between depression and disordered eating in adolescents. Eating Behaviors, 8, 440-449.

Scott, J., Chant, D., Andrews, G., y McGrath, J. (2006). Psychotic-like experiences in the general community: the correlates of CIDI psychosis screen items in an Australian sample. Psychological Medicine, 36, 231238.

Stefanis, N.C., Delespaul, Ph., Smyrnis, N., Lembesi, A., Avramopoulos, D., Evdokimidis, I., Stefanis, C.N., y Van Os, J. (2004). Is the excess risk of psychosis-like experiences in urban areas attributable to altered cognitive development? Social Psychiatry and Psychiatric Epidemiology, 39, 364-368.

Stefanis, N.C., Hanssen, M., Smirnis, N., Avramopoulos, D., Evdokimidis, I., Stefanis, C.N., Verdoux, H., y Van Os, J. (2002). Evidence that three dimensions of psychosis have a distribution in the general population. Psychological Medicine, 32, 347-358.

Svirskis, T., Korkeila, J., Heinimaa, M., Huttunen, J., Ilonen, T., Ristkari, T., Hietala, J., Syvälahti, E., McGlashan, T., Vahlberg, T., y Salokangas, R. (2007). Quality of life and functioning ability in subjects vulnerable to psychosis. Comprehensive Psychiatry, 48, 155-160.
Van Os, J., Hanssen, M., Bijl, R., y Ravelli, A. (2000). Strauss (1969) revisited: a psychosis continuum in the general population? Schizophrenia Research, 45, 11-20.

Van Os, J., Hanssen, M., Bijl, R., y Vollebergh, W. (2001). Prevalence of psychotic disorder and community symptoms. An urban-rural comparison. Archives of General Psychiatry. 58, 663-668.

Van Os, J., Hanssen, M., de Graaf, R., Vollebergh, W. (2002). Does the urban environment independently increase the risk for both negative and positive features of psychosis? Social Psychiatry and Psychiatric Epidemiology, 37, 460-464.

Van Os, J., Verdoux, H., Maurice-Tison, S., Gay, B., Liraud, F., Salamon, R., y Bourgeois, M. (1999). Self-reported psychosis-like symptoms and the continuum of psychosis. Social Psychiatry and Psychiatric Epidemiology, 34, 459-463.

Venables, P. (1995). Schizotypal personality as a developmental stage in studies for schizophrenia. En A. Raine, T. Lencz, y S. Mednick (Eds.), Schizotypal Personality (pp. 107-131). Cambridge: Cambridge University Press.

Verdoux, H., y Van Os, J. (2002). Psychotic symptoms in non-clinical populations and the continuum of psicosis. Schizophrenia Research, 54, 59-65.

Verdoux, H., Van Os, J., Maurice-Tison, S., Gay, B., Salamon, R., y Bourgeois, M. (1998). Is early adulthood a critical developmental stage for psychosis proneness? A survey of delusional ideation in normal subjects. Schizophrenia Research, 29, 247-254.

Verdoux, H., Van Os, J., Maurice-Tison, S., Gay, B., Salamon, R., y Bourgeois, M. (1999). Increased ocurrence of depression in psychosis-prone subjects: a follow-up study in primary care settings. Comprehensive Psychiatry, 40, 462-468.

Yung, A., Buck by, J., Cosgrave, E., Killackey, E., Baker, K., Cotton, S., y McGorry, P. (2007). Association between psychotic experiences and depression in a clinical sample over 6 months. Schizophrenia Research, 91, 246-253.

Yung, A., Buckby, J., Cotton, S., Cosgrave, E., Killackey, E., Stanford, C., Godfrey, K., y McGorry, P. (2006). Psychotic-like experiences in nonpsychotic help-seekers: associations with distress, depression and disability. Schizophrenia Bulletin, 32, 352-359. 